\title{
Effet de la taille de l'œuf sur le nombre de caeca pyloriques et sur la croissance précoce chez la truite arc-en-ciel.
}

\author{
J. M. BLANC, Anne-Marie ESCAFFRE, P. BERGOT
}

Station de Recherches hydrobiologiques, I.N.R.A., Saint-Pée-sur-Nivelle, 64310 Ascain, France.

Summary. Effect of egg size on pyloric caecum number and growth in juvenile rainbow trout.

Eight rainbow trout sib groups were initiated by breeding two males and four females chosen for the size heterogeneity of their ova. Before hatching, the eggs were sorted into four size grades ; batches of the hatched alevins, raised up to 6 months of age, were submitted to periodical growth measurements and to final sampling for pyloric caecum counting. The main results were:

- the number of caeca, partly determined by genetic factors, was also affected by initial egg diameter, within sib groups;

- egg size also affected alevin size, but this influence diminished with age and vanished after the 5th month. In addition, maternal variation independant from egg size was also observed during the same period, suggesting the contribution of qualitative factors :

- at 6 months of age, individual weight within the batches (and thus for relatively homogeneous egg sizes) was positively correlated with caecum number. This relationship appeared again in the batch means, mixed with a variation caused by parental origin.

\section{Introduction.}

L'effet maternel favorable inhérent à la taille des œufs sur la survie et la croissance des alevins de salmonidés a été depuis longtemps observé sur les pontes comparées de différentes femelles (Fowler, 1972 ; Gall, 1974). Plus récemment, Escaffre et Bergot (1985) ont montré chez la Truite arc-en-ciel (Salmo gairdneri Richardson) que, même à l'intérieur d'une ponte, les différences de taille des œufs sont à l'origine de variations significatives du poids chez les alevins produits.

La croissance de la truitelle arc-en-ciel apparaît par ailleurs en relation avec le nombre de ses caeca pyloriques (Bergot et al., 1981a et b). Or la variabilité du nombre de caeca, partiellement d'origine génétique additive, résulte aussi d'un effet maternel inhérent à la taille des œufs, mis en évidence chez la Truite fario (Salmo trutta L.) par Blanc et al. (1979).

Par suite, la présente étude a été effectuée dans le but d'évaluer, par triages intra-pontes, l'effet de la taille des œufs sur le nombre de caeca pyloriques et simultanément, sur la croissance précoce des alevins de Truite arc-en-ciel. 


\section{Matériel et méthodes.}

Les pontes de quatre femelles, choisies en raison de l'hétérogénéité de taille apparente de leurs ovules, ont été fécondées par deux mâles pris au hasard dans la même population, à la pisciculture de Lées-Athas (Pyrénées-Atlantiques). Les caeca pyloriques de ces reproducteurs ont été dénombrés après abattage. Après incubation, les œufs sur le point d'éclore ont été transférés à la Station de Recherches (I.N.R.A.) de Saint-Pée-sur-Nivelle.

Ils ont alors été triés selon leur diamètre, dans chacune des huit fratries, à l'aide de trois cribles faits de tiges de verre régulièrement espacées, et ayant respectivement pour intervalles de passages moyens, $3,71 \mathrm{~mm}, 3,98 \mathrm{~mm}$ et $4,25 \mathrm{~mm}$ (écart-type approximatif d'erreur de construction : 0,05 mm). Les œufs embryonnés (vivants) ont été triés et comptés dans chaque classe de taille : (1) plus de $4,25 \mathrm{~mm},(2)$ de 3,98 à $4,25 \mathrm{~mm}$, (3) de 3,71 à $3,98 \mathrm{~mm}$ et (4) moins de $3,71 \mathrm{~mm}$. Les diamètres moyens de ces classes ont été approximativement déterminés pour chaque ponte par transformation probit des distributions cumulées correspondantes (Bliss, 1967) et interpolation graphique. Ces résultats figurent dans le tableau 1.

Trois semaines après l'éclosion, 40 lots d'alevins ont été constitués pour le début de l'alimentation et l'élevage. Toutefois, compte tenu des effectifs disponibles (suite à une forte mortalité larvaire), seulement 26 lots ont pu être dotés du même effectif standard (75 individus) jugé suffisant pour un testage de croissance correct (lots A), les 14 autres lots (lots B) étant variables (20 à 70 individus). L'élevage a été réalisé dans des auges compartimentées (compartiments identiques de $80 \times 42 \mathrm{~cm}$, avec $12 \mathrm{~cm}$ de hauteur d'eau) alimentées par l'eau de la Nivelle, avec un nourrissage à satiété.

Des contrôles mensuels de croissance ont été effectués dans chaque lot entre le deuxième et le sixième mois après l'éclosion, soit par mesure de longueur (moyenne sur 20 individus), soit par pesée globale du lot. A la suite du dernier contrôle, 10 truitelles ont été sacrifiées dans chaque lot $A$ et $B$ pour dénombrement des caeca pyloriques, avec pesée de ces individus dans les lots $A$.

Les données obtenues ont été traitées par les méthodes statistiques décrites par Snedecor et Cochran (1967): Analyse de variance (en tenant compte des replications inégales selon les fratries, chapitre 16) et régression sur le diamètre des œufs (analyse de covariance, chapitre 14). Les mesures individuelles (nombre de caeca et poids) ont fait l'objet d'une transformation logarithmique préalable $\left(\log _{\mathrm{e}}\right.$ ) conformément à Bergot et al. (1981a et b).

\section{Résultats.}

a) Variation du nombre de caeca pyloriques. - Une comparaison préliminaire entre lots $A$ et lots $B$ n'ayant montré aucun effet de la densité d'élevage sur le nombre de caeca pyloriques, l'analyse a été faite sur l'ensemble des 40 lots de l'expérience. Les nombres moyens de caeca dans les différentes fratries et classes de triage des oufs sont inclus dans le tableau 1, alors que l'analyse de variance 


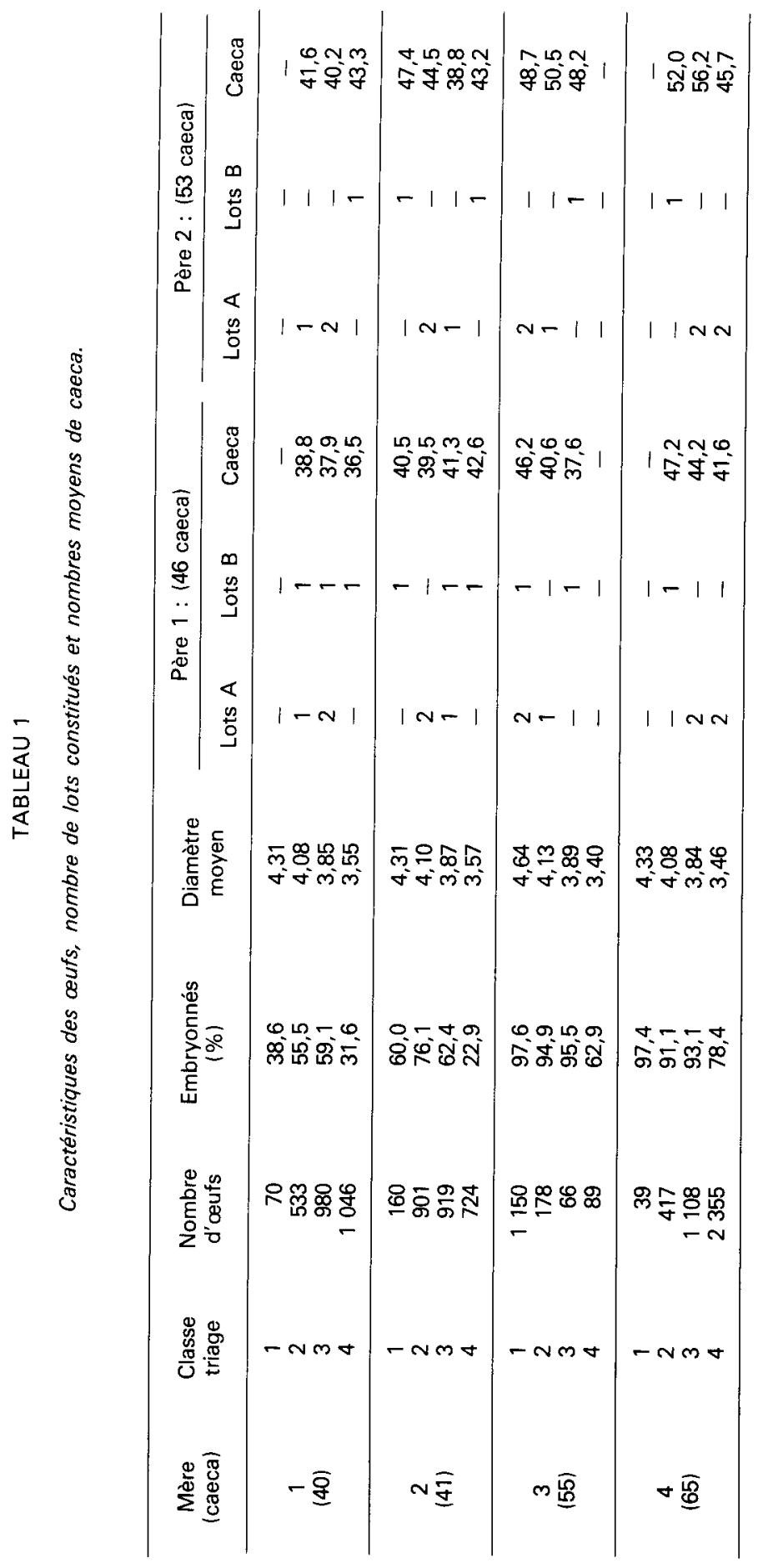


sur la variable transformée $\left(\log _{e}\right.$ ) fait l'objet du tableau 2. Ce dernier fait apparaître un effet significatif du triage des œufs, la pente de la droite de régression sur le diamètre de l'œuf $(\mathrm{mm}$ ) étant estimée à 0,12 (écart-type 0,04 avec 17 degrés de liberté) d'après les écarts entre lots intra-fratries (différences entre la moyenne de chaque lot et la moyenne de la fratrie d'où est tiré ce lot). En outre et indépendamment, on observe une variation hautement significative d'origine parentale ; la relation entre les nombres de caeca des parents et ceux de leurs progénitures apparaît à l'évidence dans le tableau 1, et conduit à estimer la pente de régression progéniture/moyenne parentale à environ 0,75 (corrélation : 0,91 ).

\section{TABLEAU 2}

Analyse de variance sur le logarithme du nombre de cacae (lots A et B).

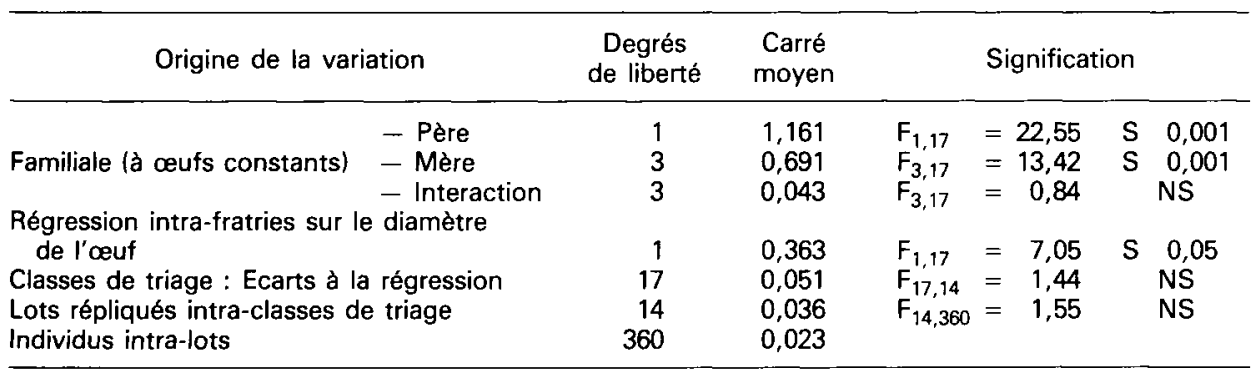

b) Effet de la taille de l'œuf sur la survie et la croissance. - Dans le tableau 1 figurent les pourcentages d'œufs embryonnés trouvés dans les différentes classes de triage : il apparaît que dans la $4^{e}$ classe (œufs inférieurs à $3,7 \mathrm{~mm}$ ), le taux d'embryonnement est réduit dans toutes les pontes alors que dans les trois autres classes, la taille des cufs ne provoque pas d'effet systématique notable. Par contre, les enregistrements ultérieurs de survie en élevage, comparés par

\section{TABLEAU 3}

Evolution de la variabilite de taille corporelle entre lots (lots A).

\begin{tabular}{|c|c|c|c|c|c|c|c|c|c|c|}
\hline \multicolumn{3}{|c|}{ Age (mois après l'éclosion) } & $\frac{1,6}{P}$ & 2,6 & \multicolumn{2}{|c|}{3,7} & \multicolumn{2}{|c|}{4,7} & \multicolumn{2}{|r|}{5,7} \\
\hline $\begin{array}{l}\text { Longueur ou P } \\
\text { Moyenne (mm } \\
\text { Régression sur } \\
\text { Variance totale }\end{array}$ & $\begin{array}{l}\text { oids } \\
\text { ou dg) } \\
\text { le diamètre des } \\
\text { entre lots } \\
\text { Liée au diamètre }\end{array}$ & ufs & $\begin{array}{l}P \\
3,45 \\
1,8 \\
0,56\end{array}$ & $\begin{array}{r}\mathrm{L} \\
44,5 \\
5,4 \\
9,9\end{array}$ & $\begin{array}{r}\mathrm{L} \\
60,8 \\
5,1 \\
18,0\end{array}$ & $\begin{array}{r}\mathrm{P} \\
30,2 \\
8,3 \\
35,1\end{array}$ & $\begin{array}{c}\mathrm{L} \\
77,5 \\
(3,3) \\
14,2\end{array}$ & $\begin{array}{c}P \\
60,2 \\
11,4 \\
69,6\end{array}$ & $\begin{array}{c}\mathrm{L} \\
88,4 \\
(5,5) \\
20,2\end{array}$ & $\begin{array}{cc} & P \\
4 & 92,1 \\
& (12,1) \\
2 & 143,3\end{array}$ \\
\hline $\begin{array}{l}\text { Composantes } \\
\text { de la variance } \\
\qquad(\%)\end{array}$ & $\begin{array}{l}\text { des œufs } \\
\text { Indépendantes }\end{array}$ & $\begin{array}{l}\text { Père } \\
\text { Mère } \\
\text { Interaction } \\
\text { Lot } \\
\text { Echantillonnage }\end{array}$ & $\begin{array}{l}69 \\
6 \\
22 \\
(0) \\
3 \\
-\end{array}$ & $\begin{array}{c}35 \\
(1) \\
56 \\
(1) \\
(-1) \\
8\end{array}$ & $\begin{array}{c}17 \\
(4) \\
64 \\
(-6) \\
12 \\
9\end{array}$ & $\begin{array}{c}23 \\
8 \\
55 \\
(1) \\
13 \\
-\end{array}$ & $\begin{array}{l}(10) \\
16 \\
52 \\
(-3) \\
(4) \\
21\end{array}$ & $\begin{array}{c}22 \\
30 \\
33 \\
(-3) \\
18 \\
-\end{array}$ & $\begin{array}{l}(18) \\
35 \\
22 \\
1-9 \\
19 \\
15\end{array}$ & $\begin{array}{c}(12) \\
42 \\
22 \\
9)(-8) \\
32 \\
-\end{array}$ \\
\hline
\end{tabular}

( 1 : estimations non significatives au seuil $p=0,05$. 
analyse de variance, ne révèlent aucun effet significatif au seuil de $5 \%$ entre classes de triage (survies moyennes: $64 \%$ du stade embryonné à l'âge de 3 semaines, $78 \%$ de 3 semaines à 6 mois).

Le tableau 3 résume les principaux paramètres statistiques qui caractérisent les longueurs et les poids enregistrés entre le $2^{e}$ et le $6^{e}$ mois dans les lots $A$ lles lots $B$ ayant généralement montré de meilleures performances en raison inverse de leurs effectifs ainsi que l'on pouvait s'y attendre). Au fur et à mesure de la croissance, la variance entre lots augmente. La taille des œufs est responsable d'une part de variation qui, en valeur absolue (mesurée par la pente de la droite de régression), augmente dans les trois premiers mois et tend à se stabiliser ensuite; en valeur relative au contraire, on observe une diminution progressive alors que d'autres sources de variation prennent de l'importance; au-delà du $5^{e}$ mois, l'effet de la taille des œufs n'est plus significatif.

A partir du $4^{\mathrm{e}}$ mois, apparaissent des variations entre lots liées à l'environnement collectif (" effet-cage "), ainsi que les effets génétiques additifs manifestés par la variation entre pères. Par contre, l'origine maternelle est responsable d'une part importante de variance dès le début de l'élevage, indépendamment de la taille des œufs ; à partir du $5^{\mathrm{e}}$ mois, la composante maternelle de variance devient du même ordre que la composante paternelle, et ne reflète donc plus que la variance génétique. On note enfin que ces effets parentaux sont additifs, puisqu'à aucun moment on n'observe d'interaction significative.

c) Relation entre caeca pyloriques et croissance. - L'analyse de variance relative aux poids des 260 individus des lots $A$ sacrifiés pour le comptage des caeca est présentée dans le tableau 4 . On observe d'abord intra-lots une corrélation poids-caeca hautement significative $(r=0,34)$, la pente de la droite de régression logarithmique correspondante étant de 0,77 lécart-type 0,14). Au niveau immédiatement supérieur (entre lots intra-fratries), bien que les différences

\section{TABLEAU 4}

Analyse de variance sur le logarithme du poids individuel à 6 mois (lots A).

Origine de la variation $\begin{gathered}\text { Degrés } \\ \text { de liberté }\end{gathered} \begin{gathered}\text { Carré } \\ \text { moyen }\end{gathered} \quad$ Signification

Entre fratries

$$
\begin{aligned}
& \text { - Père } \\
& \text { - Mere } \\
& \text { - Interaction }
\end{aligned}
$$

$1 \quad 1,309$

$3 \quad 1,362$

$3 \quad 0,122$

$\begin{array}{llll}F_{1,18} & =4,76 & S & 0,05 \\ F_{3,18} & =4,95 & S & 0,05 \\ F_{3,18} & =0,44 & \text { NS }\end{array}$

Entre lots intra-fratries

Régression sur log nombre caeca

Régression sur œuf après caeca

Ecarts à la double régression

Total

Intra-lots

Régression sur log nombre caeca

Différence entre pentes de régression

Ecarts résiduels intra-lots

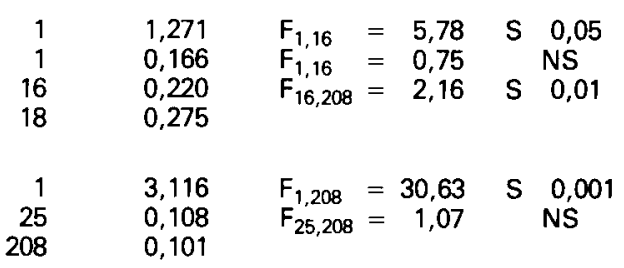


moyennes de caeca soient fortement liées aux différences de taille des œufs selon les classes de triage (tabl. 2), il apparaît ici que les caeca constituent le meilleur prédicteur du poids, leur seule contribution étant significative.

Enfin, les carrés moyens d'origines paternelle et maternelle sont significatifs (l'interaction étant inexistante), bien que l'analyse à ce niveau reste limitée faute d'un nombre suffisant de géniteurs.

\section{Discussion.}

La présente étude partage avec les travaux récents de Escaffre et Bergot (1984, 1985) l'originalité d'une méthode de triage des œufs selon leur diamètre qui permet d'apprécier les effets ultérieurs de la taille des œufs indépendamment des autres facteurs d'origine maternelle. Les lots ainsi obtenus ne sont toutefois pas parfaitement homogènes, d'une part parce que l'intervalle de classe n'est pas négligeable, et d'autre part, parce que la précision du calibrage est elle-même limitée par les imperfections de construction de l'appareil et par la forme variable et légèrement ellipsoïde des œufs. Les résultats de Escaffre et Bergot (1985) permettent d'estimer à environ 0,8 le coefficient de corrélation intra-classe relatif au poids des œufs triés selon cette méthode.

L'effet de la taille de l'œuf sur le nombre de caeca pyloriques, d'abord observé entre fratries chez la Truite fario par Blanc et al. (1979), se trouve ici mis en évidence entre classes de triage intra-fratries. Mais pour significatif qu'il soit, cet effet reste difficile à évaluer : d'une part, il ne contribue que faiblement à la variance entre lots (tabl. 2) et ceci bien que la variabilité des œufs ait été volontairement exagérée par le choix des femelles reproductrices ; d'autre part, compte tenu des imperfections du triage, une variation résiduelle de la taille des œufs intra-lots pourrait être la cause d'une petite partie de la variance résiduelle des caeca qui compte à elle seule pour les $2 / 3$ de la variance totale de ce caractère. Le principal déterminant identifié du nombre de caeca reste donc l'origine parentale, I'héritabilité ayant été antérieurement évaluée à 0,5 (Chevassus et al., 1979). Un des estimateurs de l'héritabilité est constitué par le coefficient de régression progéniture/moyenne parentale (Falconer, 1960) et la valeur obtenue dans la présente étude, malgré son imprécision, confirme l'importance du phénomène.

L'effet de la taille des œufs sur la longueur et le poids des alevins qui en sont issus a fait l'objet de multiples travaux. Tous les auteurs s'accordent sur l'importance de cet effet chez les alevins jeunes (du $1^{\mathrm{er}}$ au $3^{\mathrm{e}}$ mois) et obtiennent par comparaison entre pontes de différentes femelles, des corrélations œuf-alevin comprises entre 0,5 et 0,9 , les estimations les plus élevées correspondant aux stades les plus jeunes ; nos résultats sont ainsi en accord avec ceux de Fowler (1972) chez le Saumon chinook, de Bilton (1971) chez le Saumon sockeye, de Gall (1974) et de Chevassus (1976) chez la Truite arc-en-ciel. Mais les analyses effectuées intra-fratries par Escaffre et Bergot (1985) et dans le présent travail montrent clairement que la taille de l'œuf agit par elle-même comme un facteur environnemental, indépendamment des autres facteurs. Par suite, la notion de corrélation génétique entre œuf et alevin (Robison et Luempert, 1984) ne peut être utilisée sans précaution dans la comparaison des origines maternelles, et il est pré- 
férable de considérer cet effet maternel comme une forme d'interaction génotypemilieu, la taille des ovules étant génétiquement corrélée avec le poids corporel chez les mères (Gall, 1975).

Il est bien établi également que l'effet de la taille des œufs chez les alevins s'estompe avec l'âge. D'après Escaffre et Bergot (1985), dans le modèle simple de croissance $P=P_{0} e^{k t}$, la taille de l'œuf conditionne le poids de l'alevin au début de l'alimentation $\left(P_{0}\right)$ mais n'agit pas sur le taux de croissance ultérieur $(k)$, alors que celui-ci est soumis à l'influence des facteurs génétiques et environnementaux, notamment par le biais de la consommation alimentaire (Kinghorn, 1983). La décroissance de la contribution relative liée à la taille de l'œuf apparaît donc inéluctable, les différences d'appréciation dépendant de la puissance statistique propre à chaque expérimentation : la méthode de triage des œufs prouve son efficacité, permettant de déceler significativement la corrélation œuf-alevin jusqu'au $5^{e}$ mois, alors que les estimations de la plupart des auteurs (travaillant par comparaison de fratries) tombent en deçà du seuil de signification à partir du $4^{\mathrm{e}}$ mois. Toutefois, les recherches effectuées jusqu'à présent ne permettent pas d'apprécier l'effet secondaire que des différences de taille d'œufs peuvent avoir intra-lots sur les avantages initiaux des alevins les uns par rapport aux autres dans le cadre de la compétition alimentaire (Chevassus, 1982) : dans cette hypothèse qui reste à vérifier, la taille de l'œuf agirait ainsi indirectement sur le taux de croissance des individus élevés ensemble, produisant un effet peut être plus important et durable que celui mesuré jusqu'à présent entre lots.

Les effets maternels, enfin, ne se limitent pas à l'apport quantitatif mesuré par la taille des œufs : nos résultats font en outre apparaître dans le poids des alevins une part de variance importante d'origine maternelle, antérieure à l'extériorisation des effets génétiques additifs que la composante paternelle permet d'identifier (Falconer, 1960). Cet effet maternel indépendant de la taille des œufs suggère l'intervention de facteurs qualitatifs inhérents à ces œufs (composition des réserves vitellines, par exemple) qu'il conviendrait de préciser.

La relation entre le nombre de caeca pyloriques et la taille corporelle d'animaux de même âge a été mise en évidence (Bergot et al., 1981a et b) tant à l'intérieur de lots de plein-frères qu'entre familles. En particulier, le fait qu'une sélection paternelle sur le nombre de caeca modifie la croissance des descendants (Bergot et al., 1981b) montre l'existence d'une corrélation génétique entre les deux caractères. La question de savoir si cette corrélation témoigne d'une simple allométrie de croissance précoce ou d'une réelle relation de cause à effet par le biais de mécanismes digestifs a été discutée par ces auteurs, certains arguments étant en faveur de la seconde hypothèse. La présente étude apporte des éléments qui vont également dans ce sens, puisqu'une variation du nombre de caeca initialement liée à la taille des œufs entre lots de même origine reste corrélée à la variation de poids moyen de ces lots, alors même que l'effet direct de la taille des œufs s'estompe à ce niveau. Cette relation entre caeca pyloriques et croissance devrait susciter l'intérêt des investigateurs concernés par l'amélioration de la production piscicole. 
Remerciements. - Nous remercions Melle Huguette Poisson et M. Jean J. Yanci pour leur contribution technique à l'élevage des animaux et à la saisie des données expérimentales.

\section{Références}

BERGOT P., BLANC J. M., ESCAFFRE A. M., 1981a. Relationship between number of pyloric caeca and growth in rainbow trout (Salmo gairdneri Richardson). Aquaculture, 22, 81-96.

BERGOT P., BLANC J. M., ESCAFFRE A. M., POISSON H., 1981b. Effect of selecting sires according to their number of pyloric caeca upon the growth of offspring in rainbow trout (Salmo gairdneri Richardson). Aquaculture, 25, 207-215.

BILTON H. T., 1971. A hypothesis of alteration of age of return in successive generations of Skeena river sockeye salmon (Oncorhynchus nerka). J. Fish. Res. Bd. Canada, 28, 513-516.

BLANC J. M., CHEVASSUS B., BERGOT P., 1979. Déterminisme génétique du nombre de caeca pyloriques chez la truite fario (Salmo trutta Linné) et la truite arc-en-ciel (Salmo gairdneri Richardson). III. Effet de génotype et de la taille des œufs sur la réalisation du caractère chez la truite fario. Ann. Génét. Sél. anim., 11, 93-103.

BLISS C. I., 1967. Statistics in biology. Vol. 1, Mc Graw-Hill, New York, 558 p.

CHEVASSUS B., 1976. Variabilité et héritabilité des performances de croissance chez la truite arcen-ciel (Salmo gairdneri Richardson). Ann. Génét. Sél. anim., 8, 273-283.

CHEVASSUS B., 1982. Facteurs génétiques et sociaux influençant la croissance chez les poissons. Oceanis, 8, 579-598.

CHEVASSUS B., BLANC J. M., BERGOT P., 1979. Déterminisme génétique du nombre de caeca pyloriques chez la truite fario (Salmo trutta Linné) et la truite arc-en-ciel (Salmo gairdneri Richardson). II. Effet du génotype du milieu d'élevage et de l'alimentation sur la réalisation du caractère chez la truite arc-en-ciel. Ann. Génét. Sél. anim., 11, 79-92.

ESCAFFRE A. M., BERGOT P., 1984. Utilization of the yolk in rainbow trout alevins (Salmo gairdneri Richardson) : effect of egg size. Reprod. Nutr. Dévelop., 24, 449-460.

ESCAFFRE A. M., BERGOT P., 1985. Effet d'une alimentation précoce ou retardée sur la croissance d'alevins de truite arc-en-ciel (Salmo gairdneri) issus d'œufs de tailles différentes. Bull. Fr. Pêche Piscic., 296, 17-28.

FALCONER D. S., 1960. Introduction to quantitative genetics. Oliver and Boyd Ed. Edinburgh, $365 \mathrm{p}$.

FOWLER L. G., 1972. Growth and mortality of fingerling chinook salmon as affected by egg size. Progr. Fish Cult., 34, 66-69.

GALL G. A. E., 1974. Influence of size of eggs and age of female on hatchability and growth in rainbow trout. Calif. Fish Game, 60, 26-35.

GALL G. A. E., 1975. Genetics of reproduction in domesticated rainbow trout. J. anim. Sci., 40 , 19-28.

KINGHORN B., 1983. Genetic variation in food conversion efficiency and growth in rainbow trout. Aquaculture, 32, 141-155.

ROBISON O. W., LUEMPERT L. G., III, 1984. Genetic variation in weight and survival of brown trout (Salvelinus fontinalis). Aquaculture, 38, 155-170.

SNEDECOR G. W., COCHRAN W. G., 1967. Statistical methods. lowa State Univ. Press, Ames, $593 \mathrm{p}$. 